\title{
A Path-Loss Analysis Based WLAN Localization Method for Indoor Location Based Services
}

\author{
Jie Zhang ${ }^{+}$, Yujie Qian and Guangjie Han \\ College of IoT Engineering, Hohai University, Changzhou, China
}

\begin{abstract}
Wireless Local Area Networks (WLAN) fingerprint based indoor localization schemes have been widely studied due to the increasing requirements of Location Based Services (LBS). The feature of WLAN fingerprint is known as high accuracy and flexible adaptability, however, also high cost. To estimate the locations of wireless devices through matching their signal fingerprint, it usually requires large amount of Received Signal Strength (RSS) data from multiple Access Points (AP) to create a fingerprint database that implies a time-consuming data collection pre-process and high performance computational resources. In this article, we propose a low-cost indoor localization method which is based on environmental path-loss analysis, the method targets low accuracy room-level positioning for LBSs in large scale indoor environments.
\end{abstract}

Keywords: Wireless Local Area Networks, Indoor Localization, Path-loss, Location Based Services.

\section{Introduction}

Nowadays, we have witnessed the rapid increase of indoor Location Based Services (LBS) for convenient of human life, however we also see the widely-used Global Positioning System (GPS) is not working well in indoor environments. Hence, with the increase of Wireless Local Area Networks (WLAN) deployed in the public, WLAN signal based indoor localization techniques are widely studied to reach the positioning requirements in various LBSs ${ }^{[1-3]}$. Compare to the GPS localization which measures absolute coordinates of wireless devices, WLAN based indoor localization is a method that measures relative coordinates from some particular reference points, such as WLAN Access Points (AP).

WLAN based localization has higher accuracy than GPS localization in indoor environment, and also has flexible adaptability since the WLAN has become a universal civilian network. However, it also faces technical difficulties because of the various environmental factors. For examples, WLAN triangulation requires measuring signal distances among end user and neighbouring APs ${ }^{[4][5]}$, and the complex indoor environment causes various path-loss exponent and inaccurate distance measurement; WLAN fingerprint ${ }^{[6-8]}$ requires creating a Received Signal Strength (RSS) distribution database to map the end user's signal pattern, and higher environmental complexity requires larger database and longer data collection pre-process.

In this article, we propose a path-loss analysis based WLAN localization method (PLWL). The method is motivated by WLAN fingerprint method, and aims to reduce the temporal and computational cost from the RSS database creation. The PLWL calculates the path-loss exponent by measuring RSS at particular indoor locations, and then create a database which includes the variations of path-loss in different rooms. After that, calculates moving end's path-loss variations by measuring RSS, and finally analyses the similarly among each databases to locate its relative coordinate. The output of PLWL is not an accurate coordination but a very accurate room unit, because the contribution of this article targets the room-level localization, which is the most commonly appeared indoor LBS requirements.

\footnotetext{
+ Corresponding author. Tel.: + 8618261156629 ; fax: + 8651985287122
}

E-mail address: $20141931 @$ hhu.edu.cn 


\section{Related Works}

Typical WLAN positioning techniques include triangulation and fingerprint. Triangulation is based on geometrical calculations (Fig. 1), and the key is the estimation of the signal distance between transmitter and receiver ${ }^{[9][10]}$. The typical methods which are used for estimating distance include RSS measurement, Time of Arrival (TOA), Time Difference of Arrival (TDOA) and Angle of Arrival (AOA), and the common problem in indoor localization is that irregular environment causes inaccurate distance measurement ${ }^{[11]} . \operatorname{In}^{\text {[9] }}$, $\mathrm{Z}$. Li et al. related the filtered signal power information to signal propagation range to measure the signal distance; G. Shan et al. ${ }^{[10]}$ targeted a Bluetooth based triangulation and presented a 3-dimensional triangulation scheme compare to traditional 2-dimensional triangulation to minimize the distance error. The 3 -dimensional localization technique was carefully introduced in ${ }^{[11]}$, which uses four or more reference point to localize an unknown point.

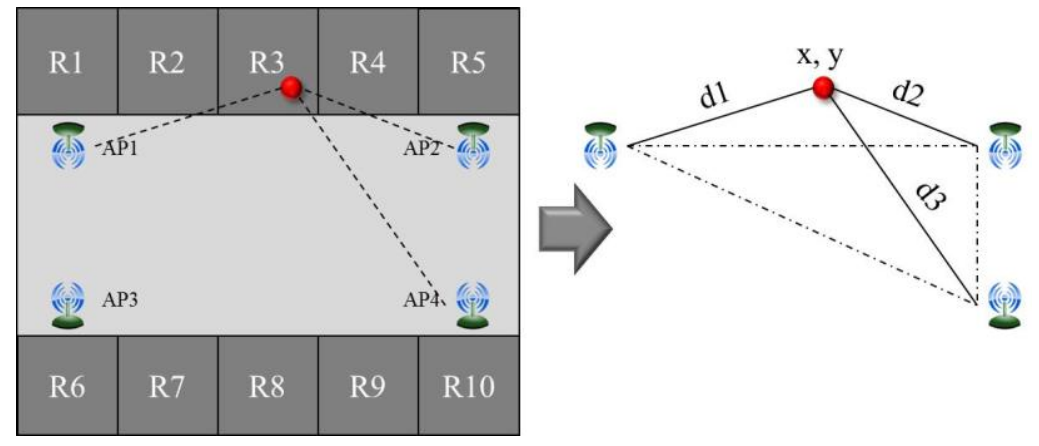

Fig. 1: An example of WLAN triangulation based indoor localization.

Fingerprint method is based on data analysis; first it measures RSS information from reference stations such as AP, and then creates a RSS map and related reference database to localize a new set of RSS for a point (Fig. 2). The focused issue in the WLAN fingerprint is the cost efficiency of time and computational resources ${ }^{[12][13]}$ since the shortcoming is known as high time and labor cost. J. Niu et al. ${ }^{[12]}$ used crowdsourcing to automatically collect the fingerprints, and the authors also proposed a ZigBee assisted indoor localization which uses ZigBee interfaces to collect mixed WiFi signals and create a fingerprint database ${ }^{[13]}$.

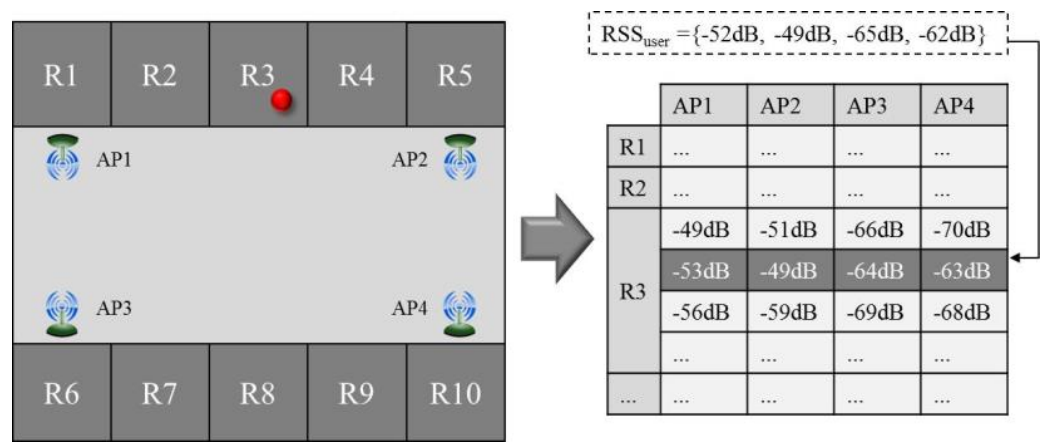

Fig. 2: An example of WLAN fingerprint based indoor localization.

\section{Path-loss Analysis Based WLAN Localization}

The proposed PLWL is described in this section. The PLWL inherits WLAN fingerprint where uses a reference database to locate a specific end user. The difference is, the database is created by information of path-loss exponent (PLe) in particular areas. The PLe is calculated by the signal distance estimation method, which is a key part of the WLAN triangulation method. Equation (1) is a signal distance calculation equation using the RSS information, which is converted from ${ }^{[14]}$.

$$
d=10^{\frac{P L-c-s}{10 n}}
$$

Where $d$ is the distance between sender and receiver, $n$ is the PLe, $P L$ is the path-loss value in $\mathrm{dB}$ equals to Transmission Power-RSS, $c$ is a pre-measured path-loss value in a certain distance (normally 1 meter), $s$ is the standard deviation of shadow fading which recommended as 3-7 $\mathrm{dB}$ in ${ }^{[13]}$.

From (1), we can calculate the PLe as: 


$$
n=\frac{P L-c-s}{10 \log d}
$$

Our proposal is under an assumption that the target end system can detect its movement. When the target moves, it triggers the PLWL and sends the RSS information to the localization server, then the server analyse the RSS variation and find a mapping PLe variation from the database. Follows are detailed descriptions of the PLWL processes.

\subsection{Creating Path-loss Exponent Database}

Fig. 3 illustrates the data collection and database creation process.

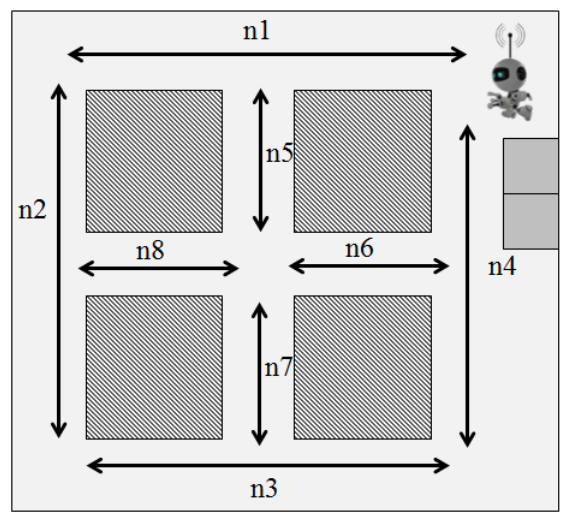

(a)

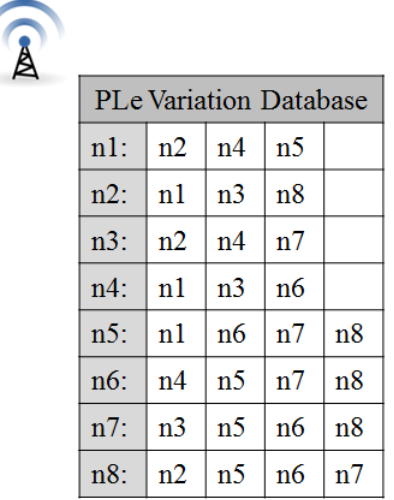

(b)

Fig. 3: An example of collecting PLes and creating localization database.

As in Fig. 3(a), an autonomic robot which has a WLAN interface and a function of avoiding barriers will start from an AP and then move into a room, while moving, it sends RSS, moved distance and barrier information (turning event) to the AP, and then the server calculates PLe with the information. The PLe is calculated and averaged continuously until the robot met a barrier and turned to other direction, and then the pre-measured PLe will be stored into database and a new PLe measurement will be started. By this, the database includes all of the PLes with different barrier is created. Fig. 3(b) shows the database, it uses the robot's turning events to store the PLe information by pairs since the localization method is finding the PLe variations, for example, $\mathrm{n} 1$ has 3 pairs which are $\mathrm{n} 1: \mathrm{n} 2, \mathrm{n} 1: \mathrm{n} 4$ and $\mathrm{n} 1: \mathrm{n} 5$.

\subsection{Path-loss Analysis Based Indoor Localization}

After creating the PLe database, the indoor localization can be performed as Fig. 4.

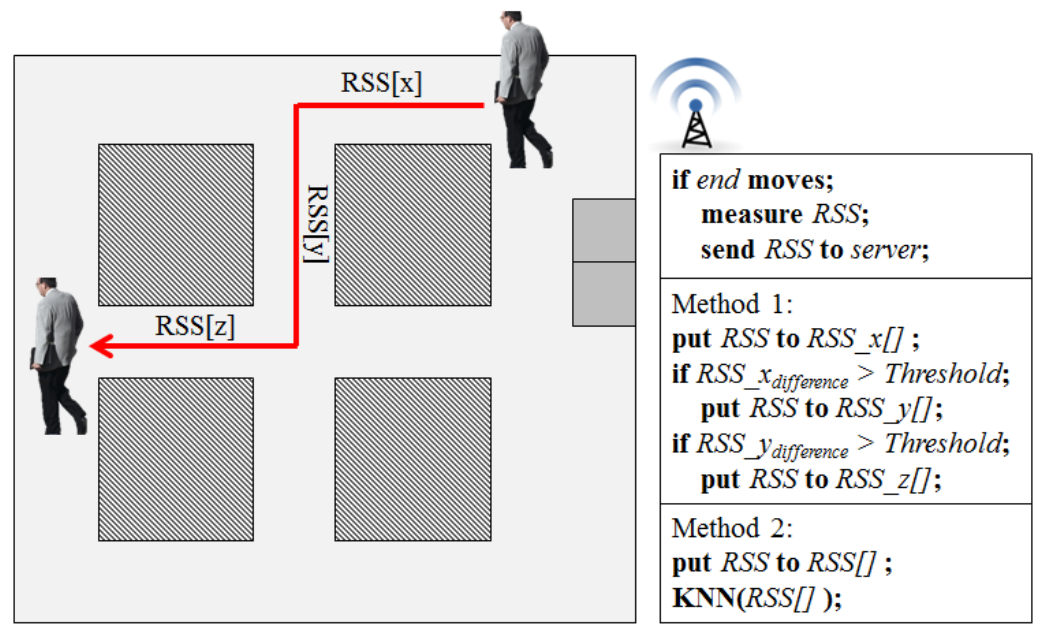

Fig. 4: An example of PLWL.

First, the end user's wireless device (e.g. Smartphone) detects if the use is moving, if is, measure the RSS and send to local AP (server). At the server side, it needs to classify the end's moving areas to evaluate PLe variation. There are two methods to classify the areas: The first one is observing RSS variation, if the 
RSS variation is suddenly changed, it means the end user may meet a barrier and make a detour, and then the previously collected RSS can be classified as from same moving area; second one is using data analysis algorithms, for example, collecting RSS in a certain period and then use the $k$-Nearest Neighbour (kNN) clustering to classify the moving areas. First one is more correct in the classification, since the number of moving areas can be exactly identified; however the latter one can save resources, because it does not require monitoring the RSS variation.

Finally, the end user's location is evaluated by following algorithm1:

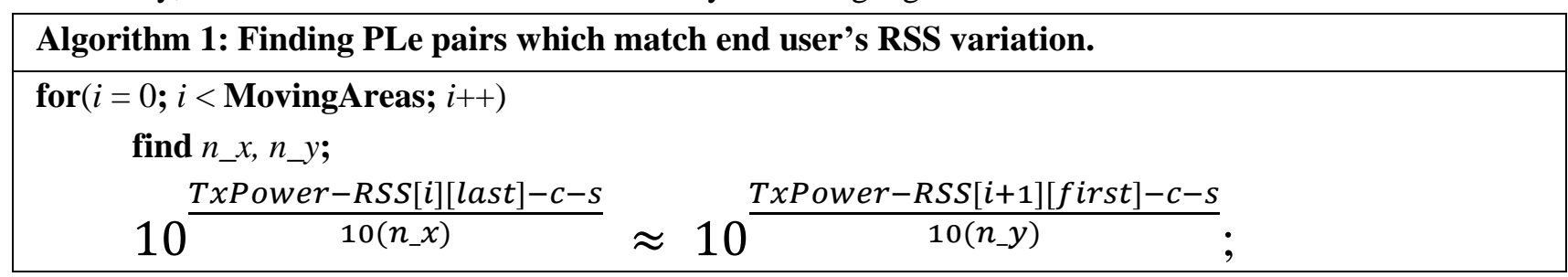

Algorithm 1 targets two time points of an end user is leaving a moving area and then entering another moving area, which should have similar signal distance from AP. With this, server will search the database to find a room which has most matching PLe pairs.

\section{Conclusion and Further Works}

In this article, we proposed a path-loss analysis based indoor localization Method named PLWL, the method is an extension of WLAN fingerprint based localization which also used the distance and path-loss evaluation from WLAN triangulation. The proposed method targets LBSs which require only room-level localization, and attempts to solve the high cost problem in WLAN fingerprint based localization. Yet the research is in theoretical phase, for the next works in few months, we intend to design and perform the experiment of creating PLe database in several indoor environments. After that, with the experimental data we will simulate the PLWL in large scale indoor environments and then compare it with traditional localization methods to prove the efficiency and practicality.

\section{Acknowledgements}

The work is supported by National Natural Science Foundation of China (No. 61601169, No. 11604077) and Jiangsu Provincial Natural Science Foundation for Youths (No. BK20150248).

\section{References}

[1] M. Zhou, F. Qiu, Z. Tian, K. Xu, Q. Jiang. Positioning Error vs. Signal Distribution: An Analysis Towards Lower Error Bound in WLAN Fingerprint Based Indoor Localization. 2015 IEEE Global Communications Conference (GLOBECOM). San Diego. 2015, pp. 1-6.

[2] J. Qiu, X.Wang, and G. Dai. Improving the Indoor Localization Accuracy for CPS by Reorganizing the Fingerprint Signatures. Int. J. Distrib. Sens. N. 2014, Article ID 415710: 1-13.

[3] J. Koo, H.Cha. Unsupervised Locating of WiFi Access Points Using Smartphones. IEEE. T. Syst. Msn. Cy. C. 2012, 42(6):1341-1353.

[4] Skyhook. Location-based services that choose location algorithms based on number of detected access points within range of user device. US Patent 7305245. 2007

[5] Skyhook. Methods and systems for estimating a user position in a WLAN positioning system based on user assigned access point locations. US Patent 7471954. 2008

[6] P. Bahl and V. N. Padmanabhan. RADAR: An In-Building RF-based User Location and Tracking System. Proc. Of IEEE INFOCOM. Tel Aviv. 2000, pp. 775-784.

[7] M. Zhou, F. Qiu, Z. Tian, H. Wu, Q. Zhang and W. He. An Information-Based Approach to Precision Analysis of Indoor WLAN Localization Using Location Fingerprint. ENTROPY. 2015, 17(12): 8031-8055.

[8] J. Torres, O. Belmonte, R. Montoliu, S. Trilles and A. Calia. How Feasible Is WiFi Fingerprint-Based Indoor Positioning for In-Home Monitoring?. 2016 12th International Conference on Intelligent Environments (IE). London. 2016, pp. 68-75. 
[9] Z. Li, T. Braun, D. C. Dimitrova. A Passive WiFi Source Localization System based on Fine-grained Power-based Trilateration. Pro. of the WoWMoM 2015: A World of Wireless Mobile and Multimedia Networks. Boston. 2015.

[10] G. Shan, B. Park, S. Nam, B. Kim, B. Roh and Y. Ko. A 3-Dimensional Triangulation Scheme To Improve The Accuracy of Indoor Localization for IoT Services. IEEE Pacific RIM Conference on Communications, Computers, and Signal Processing. Victoria. 2015, pp. 359-363.

[11] J. Li , X. Zhong and I. Lu. Three-Dimensional Node Localization Algorithm for WSN Based on Differential RSS Irregular Transmission Model. J COMMUN. 2014, 9(5): 391-397.

[12] J. Niu, B. Wang, L. Cheng and J. J. P. C. Rodrigues. WicLoc: An Indoor Localization System based on WiFi Fingerprints and Crowdsourcing. 2015 IEEE International Conference on Communications (ICC). London. 2015, pp. 3008-3013.

[13] J. Niu, B. Wang, L. Shu, T. Q. Duong, Y. Chen. ZIL: An Energy-Efficient Indoor Localization System Using ZigBee Radio to Detect WiFi Fingerprints. IEEE. J. Sel. Area. Comm. 2015, 33(7): 1431-1442.

[14] Cisco. Location Tracking Approaches. WiFi location-based services 4.1 design Guide. 2008. 